\title{
Optimization of Technological Parameters of Water Jet Cutting of Red Oak and Bamboo Based on Three- Dimensional Surface Topography Measurement
}

\author{
Wenbo Xie, ${ }^{a}$ Jiujin Fang, ${ }^{\mathrm{b}}$ Zheng Wang, ${ }^{\mathrm{a}, *}$ and Lingxiu Huang ${ }^{\mathrm{b}}$ \\ In order to accurately and reliably optimize the process parameters of ultra- \\ high pressure water jet cutting for wood, water jet processing was conducted \\ on red oak and bamboo floor specimens, and the materials were scanned for \\ three-dimensional surface topography. The surface roughness value of the cut \\ surface of the test piece was measured by the orthogonal measurement \\ method as an evaluation index to determine the optimal arrangement of the \\ influencing factors and the process parameters. Finally, the water cutting \\ mechanism and three-dimensional shape of the processed test piece were \\ analyzed. The factors affecting were as follows: cutting pressure $>$ feeding \\ speed $>$ abrasive flow rate $>$ target distance $>$ air-dry density. The optimal \\ experimental scheme included an abrasive flow rate of $35 \mathrm{~kg} / \mathrm{h}$, cutting \\ pressure of $310 \mathrm{kPa}$, feed speed of $250 \mathrm{~mm} / \mathrm{s}$, target distance of $3 \mathrm{~mm}$, and \\ air dry density of $620 \mathrm{~kg} / \mathrm{m}^{3}$. Under these processing conditions, the surface \\ roughness values of water jet red oak and bamboo specimens were relatively \\ small, and the processing quality was the best. The results of the above- \\ mentioned research can provide a reference for the research and application \\ optimization design of wood and bamboo processing for ultra-high pressure \\ water jet technology.
}

Keywords: Ultra-high pressure water jet; Red oak and bamboo; Surface roughness; Three-dimensional Surface topography; Process parameters; Cutting mechanism

Contact information: a: College of Materials Science and Engineering, Nanjing Forestry University, Nanjing, Jiangsu 210037, China; b: College of Mechanical and Electronic Engineering, Nanjing Forestry University, Nanjing, Jiangsu 210037, China; *Corresponding author: wangzheng63258@163.com

\section{INTRODUCTION}

Ultra-high pressure water flow cutting technology is a new comprehensive processing technology that has matured in the past 30 years and conforms to the concept of green environmental protection. It has many advantages; the technology is incomparable (Li et al. 2018a). In the 1980s, abrasive water jets were used for cutting materials for the first time and have been found to be ideal tools for cutting a wide variety of materials. The use of ultra-high pressure water jet technology to process precious wood has obvious advantages such as excellent cutting quality, high efficiency, low cost, environmental protection, and simple operation of the system, which can reasonably improve the utilization rate of wood (Cao et al. 2016; Li et al. 2018b). Wood has anisotropy and complexity. Considering the influence of pressure, target distance, and feed rate on the quality of wood cutting, it is more complicated than the cutting process of isotropic materials (Li 2015; Liu et al. 2018).

He et al. (2008) designed a four-cylinder interlocking pneumatic clamping device matched with the SQ-WJG40 high-pressure water jet cutting machine. Wang et al. (2010) 
analyzed the effects of parameters such as wood dry density, water cutting pressure, feed rate, garnet grain size, and cutting thickness on the surface roughness of the machine by testing the common tropical woods of the three types of flooring; water cutting pressure has the greatest impact on test results. Cao et al. (2016) used the orthogonal test method to carry out water jet cutting tests on medium density fiberboard and Yiyang solid wood specimens. When the air-drying density is $0.387 \mathrm{~g} / \mathrm{cm}^{3}$, the pressure is $300 \mathrm{MPa}$, the feed rate is $1,000 \mathrm{~mm} / \mathrm{s}$, the target distance is $5 \mathrm{~mm}$, and the sand adjustment speed is $20 \mathrm{~kg} / \mathrm{h}$, the processing surface roughness is the smallest, and the processing quality is the best. Barcík et al. (2011) studied the MDF process of abrasive water jet cutting and the influence of technical parameters, material parameters, and cutting angle on the cutting width of the material. Within the reasonable use of technical parameters, water jet cutting can be preferentially applied to composite processing; when the water jet cuts the longitudinal cutting material, the slit width becomes smaller. Narayanan et al. (2013) developed a threephase flow phenomenological model in the nozzle of water jet abrasive cutting and verified the relevant tests. The results show that the correlation between the test model and the test results exceeds $95 \%$. At present, research on water cutting is aimed at isotropic materials including rock and ceramics and wood composite materials, such as fiberboard. To realize the modern production of wood crafts, it is important to optimize the design of ultra-high pressure water jet cutting process (Ketan et al. 2019; Miao et al. 2019; Pahuja and Ramulu 2019; Zhang et al. 2019).

Past research on the optimization of process parameters for ultra-high pressure water jet cutting wood showed that the surface roughness of wood and bamboo affects its appearance, surface finish, gluing, and other qualities. The surface roughness value of the cut test piece is used as a quality evaluation index. Therefore, it is important to select suitable surface roughness methods and means for measuring anisotropic materials such as wood, in order to obtain accurate and reliable surface roughness values after processing. In current research, the stylus profile method is used often to measure the surface roughness of materials. Wood is a porous anisotropic material, and its surface roughness is more complex than isotropic materials. Therefore, when the surface roughness of wood is measured by the stylus type contour method, the diameter of the tip of the stylus and the positioning and scribing measurement on the processing surface of the material is likely to cause large measurement errors. Because the computer vision inspection technology of the optical method is more suitable for the detection of wood surface roughness, this paper uses the scanning probe type three-dimensional surface topography method based on the principle of optical lever method to measure red oak specimens and bamboo specimens surface roughness. These experiments were used to determine the order of the influencing factors of the water cutting test piece and the optimal plan of the process parameters. The water jet cutting processing mechanism was analyzed by three-dimensional surface topography. The results provide support for the ultra-high pressure water jet technology in wood processing research.

\section{EXPERIMENTAL}

\section{Materials and Methods}

Red oak (Quercus spp.) of size $1050 \mathrm{~mm} \times 120 \mathrm{~mm} \times 20 \mathrm{~mm}$, air-dry density of $620 \mathrm{~kg} / \mathrm{m}^{3}$, and moisture content of $8 \%$ was produced in the northern United States. Bamboo (Phyllostachys heterocycla (Carr.) Mitford cv. pubescens) flooring with 
dimensions of $900 \mathrm{~mm} \times 90 \mathrm{~mm} \times 15 \mathrm{~mm}$, air-dry density of $528 \mathrm{~kg} / \mathrm{m}^{3}$, and moisture content of $12 \%$ was produced in Zhejiang Dazhuang Industrial Group Co., Ltd. (Hangzhou, China). Garnet sand (60 mesh) was produced in Nantong, Jiangsu, China.

\section{Test Instruments}

Experiments were performed with a WC40WA1312H numerical control ultra-high pressure water jet cutting machine (Nanjing Analyzer Software Engineering Co., Ltd., Nanjing, China) composed of an SQ-WJG44 ultra-high pressure water jet generator, gantry type numerical control table, jet cutting system, and numerical control operating system. The water jet generator consisted of a system of pressurization, water supply, pressurized constant pressure, nozzle line, water collection, and water circulation treatment, with a maximum water pressure of $300 \mathrm{MPa}$. The numerical control operating system consisted of a standard industrial control machine, an independent control cabinet, and a dedicated CAD/CAM system. The workflow is shown in Fig. 1.

The scanning probe-type three-dimensional surface topography measuring instrument consisted of a laser adjusting mirror, photosensitive device, high-precision three-axis scanning table, probe, and camera. The scanning accuracy was $0.5 \mu \mathrm{m}$.

The GDS-100 constant temperature and humidity test chamber used computer temperature control humidity and saturated gas humidification. The temperature range was $-70{ }^{\circ} \mathrm{C}$ to $+100{ }^{\circ} \mathrm{C}\left(+150{ }^{\circ} \mathrm{C}\right)$, and the humidity range was $38 \%$ to $98 \%$ relative humidity (RH). An HK-30 wood moisture meter was procured from Shenzhen Jumaoyuan Science and Technology Co., Ltd. (Shenzhen, China).

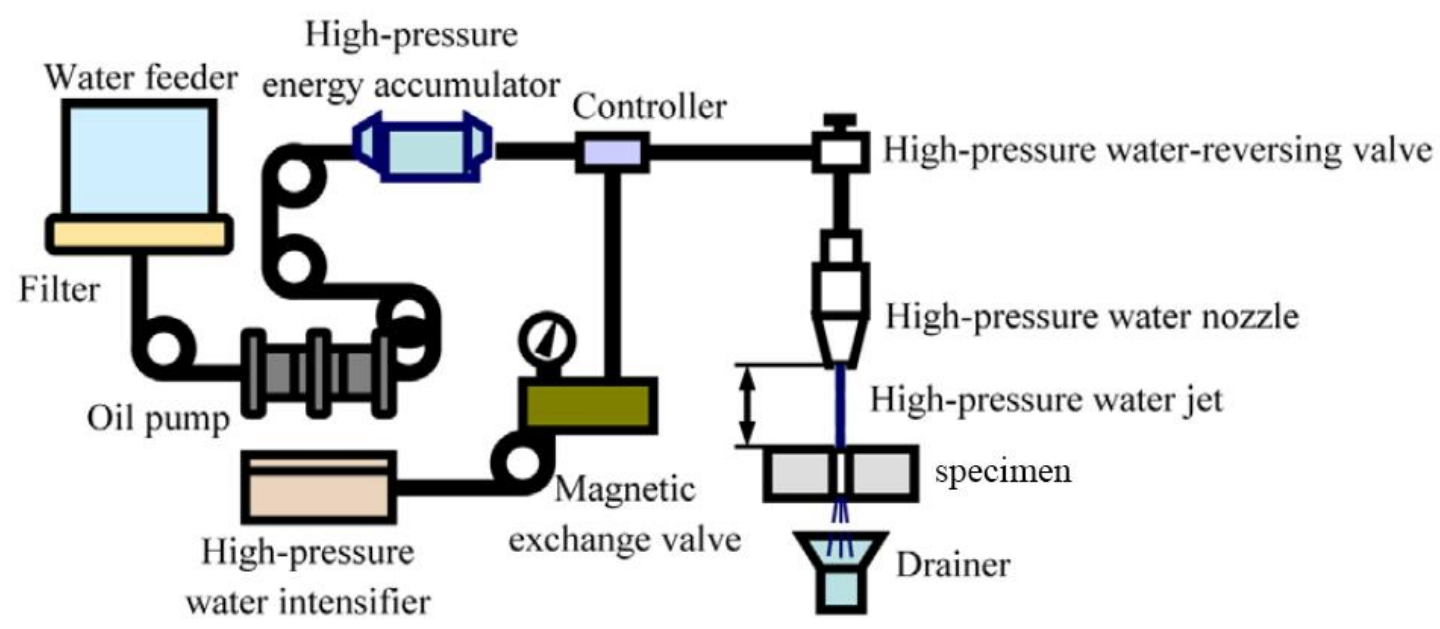

Fig. 1. UHP water jet cutting rosewood process workflow

\section{Experiment Design and Process}

An orthogonal analysis table $\mathrm{L}_{8}\left(2^{5}\right)$ was adopted in the water jet test of red oak and bamboo flooring. The test factors included the abrasive flow rate, water jet pressure, target distance, feed rate, and air-dry density, with 2 levels for each factor (Table 1).

The specimens are shown in Fig. 2. The main processing sequence was CAD pattern design; test piece fixation; sand adjustment; setting cutting pressure and feed speed; adjusting target distance; positioning cutting; drying treatment; and three-dimensional surface topography to measure cutting surface roughness. 
Table 1. Orthogonal Test Table of Water Jet

\begin{tabular}{|l|c|l|l|l|c|}
\hline \multirow{2}{*}{ Test Level } & $\begin{array}{c}\text { Abrasive } \\
\text { Flow Rate (A) } \\
(\mathrm{kg} / \mathrm{h})\end{array}$ & $\begin{array}{c}\text { Pressure (B) } \\
(\mathrm{MPa})\end{array}$ & $\begin{array}{c}\text { Feed Rate } \\
(\mathrm{C}) \\
(\mathrm{mm} / \mathrm{s})\end{array}$ & $\begin{array}{c}\text { Target } \\
\text { Distance (D) } \\
(\mathrm{mm})\end{array}$ & $\begin{array}{c}\text { Air-dry } \\
\text { Density } \\
(\mathrm{E}) \\
\left(\mathrm{kg} / \mathrm{m}^{3}\right)\end{array}$ \\
\hline 1 & 35 & 250 & 250 & 3 & 620 \\
\hline 2 & 25 & 310 & 350 & 8 & 528 \\
\hline
\end{tabular}

A scanning probe-type three-dimensional surface topography measuring device composed of a laser, a first mirror, a second mirror, a Charge-coupled Device, a controller, a stepper motor, a table, and a probe was used in this research. The test piece is placed on a table driven by a stepper motor, which can be lifted and translated. Through the probe that is in contact with the surface of the test piece, the topography and surface roughness of the entire surface of the test piece are measured.

The cutting numbers of the red oak specimens were $\mathrm{X} 1, \mathrm{X} 3, \mathrm{X} 6$, and $\mathrm{X} 8$; the cutting numbers of the bamboo flooring specimens were Z2, Z4, Z5, and Z7.

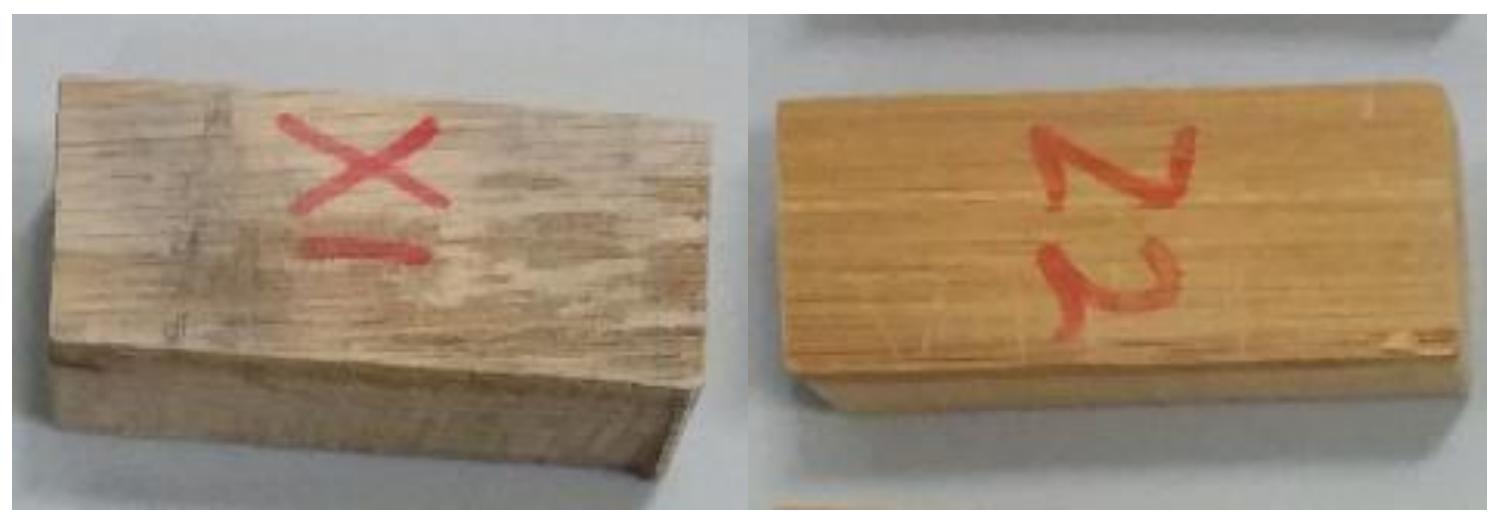

Fig. 2. Processing specimen

\section{RESULTS AND DISCUSSION}

\section{Surface Roughness Measurement and Analysis}

The results of surface roughness measurement and its orthogonal test analysis are shown in Table 2. The $R_{\mathrm{a}}$ value is the preferred metric, according to GB/T 1031 (2009). The test influencing factors of the abrasive jet were ranked as BCADE; that is, pressure > feed rate $>$ abrasive flow rate $>$ target distance $>$ air-dry density. The optimal test plan should include an abrasive flow rate of $35 \mathrm{~kg} / \mathrm{h}$, cutting pressure of $310 \mathrm{kPa}$, feed rate of $250 \mathrm{~mm} / \mathrm{s}$, target distance of $3 \mathrm{~mm}$, and air-dry density of $620 \mathrm{~kg} / \mathrm{m}^{3}$. The surface roughness of the cut material was relatively small under this processing. The quality of the processed materials was the best. The reduction in abrasive flow rate and pressure resulted in reduced erosion capacity and kinetic energy of the cut. The increase in feed rate and the increase in target distance increased the angle of the abrasive jet, resulting in an increase in the surface area of the wood. The integrity of the wood surface treated under high pressure water flow was reduced. These reasons led to the greatest surface roughness. 
Table 1. Orthogonal Design Analysis Table $L_{8}\left(2^{5}\right)$ of Abrasive Jet

\begin{tabular}{|c|c|c|c|c|c|c|}
\hline \multirow{2}{*}{ NO. } & \multicolumn{4}{|c|}{ Factor } & & \multirow{2}{*}{$\mathrm{Ra}(\mu \mathrm{m})$} \\
\hline & $A$ & $B$ & C & $\mathrm{D}$ & $E$ & \\
\hline $1(\mathrm{X} 1)$ & 1 & 1 & 1 & 1 & 1 & 3.74 \\
\hline $2(Z 2)$ & 1 & 1 & 1 & 2 & 2 & 5.19 \\
\hline $3(\mathrm{X} 3)$ & 1 & 2 & 2 & 1 & 1 & 4.79 \\
\hline $4(Z 4)$ & 1 & 2 & 2 & 2 & 2 & 4.01 \\
\hline $5(Z 5)$ & 2 & 1 & 2 & 1 & 2 & 6.29 \\
\hline $6(X 6)$ & 2 & 1 & 2 & 2 & 1 & 5.89 \\
\hline $7(\mathrm{Z7})$ & 2 & 2 & 1 & 1 & 2 & 3.40 \\
\hline $8(X 8)$ & 2 & 2 & 1 & 2 & 1 & 3.89 \\
\hline $\mathrm{K} 1$ & 17.73 & 21.11 & 16.22 & 18.22 & 18.31 & \\
\hline K2 & 19.47 & 16.09 & 20.98 & 18.98 & 18.89 & \\
\hline $\mathrm{k} 1$ & 3.55 & 4.22 & 3.24 & 3.64 & 3.66 & \\
\hline k2 & 3.89 & 3.22 & 4.20 & 3.80 & 3.78 & \\
\hline Range & 0.35 & 1.00 & 0.95 & 0.15 & 0.12 & 2.89 \\
\hline $\begin{array}{l}\text { Optimal } \\
\text { scheme }\end{array}$ & $\mathrm{A} 1$ & B2 & C1 & D1 & E1 & \\
\hline
\end{tabular}

\section{D Surface Topography Analysis}

Table 2 and Fig. 4 show that the surface roughness value of the $\mathrm{Z} 7$ test piece was the smallest, and the surface roughness value of the Z5 test piece was the largest. Comparing the surface roughness values of the Z7 and Z5 specimens with the corresponding three-dimensional surface topography measurement results of Fig. 4, the Z5 specimen had a poor overall surface cutting effect compared with the Z7 specimen, and there were many color differences on the Z5 diagram region. The cutting pressure of the $\mathrm{Z} 5$ test piece was smaller than that of the $\mathrm{Z7}$ test piece, and the feed speed was higher than that of the $\mathrm{Z7}$ test piece, resulting in a relatively small cutting kinetic energy and an increase of the cutting angle, so that the Z5 surface was not completely eroded during the water cutting process and the moving process was continued. The result was that the surface roughness of the Z5 specimen was the largest. Similarly, the cutting pressure and feed rate of the $\mathrm{Z7}$ specimens in the most influential factors satisfied the optimal conditions, so the cutting effect of the $\mathrm{Z7}$ specimen was the best among all the specimens.

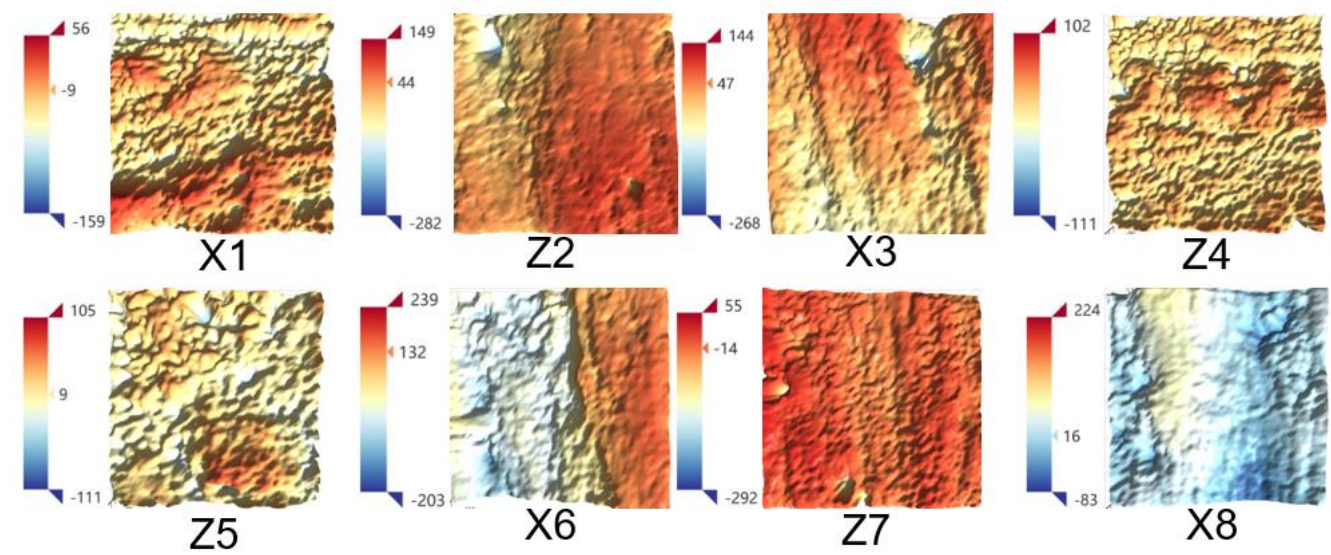

Fig. 4. Three-dimensional surface topography 
The surface roughness of the $\mathrm{X} 1$ test piece was smaller than that of the $\mathrm{Z} 2$ test piece, and the color range of the X1 test piece reflected in Fig. 2 was small. The X1 test piece was red oak. Under the same conditions of abrasive flow rate, pressure, and feed rate, the target distance used in the orthogonal test was small. Thus, the kinetic energy loss was small, and the cutting effect was good.

The X3 test piece was red oak with an $R_{\mathrm{a}}$ of $4.79 \mu \mathrm{m}$. As shown in Fig. 4, the color range of the $\mathrm{X} 3$ test piece was increased because the water jet pressure was increased. The initial damage zone range was increased, and the feed speed was increased, resulting in incomplete cutting.

The $R_{\mathrm{a}}$ of the Z4 specimen was $4.01 \mu \mathrm{m}$, which was smaller than the surface roughness of the $\mathrm{Z} 2$ specimen, and the three-dimensional topography was more uniform. The main reason was that the higher cutting pressure results in a more complete cutting surface.

In Fig. 4 , the X6 test piece $\left(R_{\mathrm{a}}=5.89 \mu \mathrm{m}\right)$ was rougher than the three-dimensional topography of the $\mathrm{X} 8$ test piece $\left(R_{\mathrm{a}}=3.89 \mu \mathrm{m}\right)$. The main reason was that the cutting pressure was low and the feed rate was fast, resulting in a faster feed rate. The cutting kinetic energy was relatively small, and the cutting angle was increased. Therefore, the water cutting did not completely erode the X6 surface and continued to move so that the surface roughness of the X6 test piece was relatively large. Combined with the processing conditions of X6 specimens and X1 and X8 specimens, the difference was the feed rate, that is, the feed rate had a greater impact on the red oak water cutting experiment.

Under the conditions determined by each process parameter of the orthogonal test, the kinetic energy of the water jet cutting material showed a trend of high to low with the thickness from shallow to deep.

\section{CONCLUSIONS}

1. The factors affecting the water cutting test of red oak and bamboo specimens are ranked as follows: cutting pressure $>$ feed rate $>$ abrasive flow rate $>$ target distance $>$ air-dry density. The optimal test process scheme was an abrasive flow rate of $35 \mathrm{~kg} / \mathrm{h}$, cutting pressure of $310 \mathrm{kPa}$, feed rate of $250 \mathrm{~mm} / \mathrm{s}$, target distance of $3 \mathrm{~mm}$, and airdry density of $620 \mathrm{~kg} / \mathrm{m}^{3}$. Under these processing conditions, the surface roughness values of water-cut red oak and bamboo specimens were relatively small, and the quality of the processed materials was the best.

2. Based on the three-dimensional surface topography method, the surface roughness of water-cut red oak and bamboo specimens can be measured, and the influencing factors of water-cut specimens can be reliably determined, and the optimal scheme of process parameters can be obtained.

3. The measured surface roughness of the $Z 7$ bamboo specimen achieved the minimum value $(3.4 \mu \mathrm{m})$, and the surface roughness of the $\mathrm{Z} 5$ bamboo specimen exhibited the maximum value $(6.29 \mu \mathrm{m})$. The cutting pressure of the $Z 5$ test piece was smaller than that of the $\mathrm{Z7}$ test piece, and the feed speed was larger than that of the $\mathrm{Z7}$ test piece, resulting in a relatively small cutting kinetic energy and an increase in the cutting angle, so that the Z5 surface was not completely washed during the water cutting process and it continues to move during processing, eventually leading to the maximum surface roughness of the Z5 specimen. 
4. The surface roughness of X1 $(3.74 \mu \mathrm{m})$ in redwood was the minimum, and the surface roughness of X6 $(5.89 \mu \mathrm{m})$ was the maximum, and the feed rate had a great effect on the water cutting experiments of red oak.

\section{ACKNOWLEDGMENTS}

This study was funded by the 2018 Postgraduate Research \& Practice Innovation Program of Jiangsu Province (KYCX18_0963).

\section{REFERENCES CITED}

Barcík, Š., Kvietková, M., Kminiak, R., and Aláč, P. (2011). “Optimization of cutting process of medium density fibreboards by the abrasive water-jet," Drvna Industrija 62(4), 263-268. DOI: 10.5552/drind.2011.1113

Cao, Y., Wang, Y. L., Gao, Z. Z., and Wang, Z. (2016). "Wooden crafts processing parameter optimization and analysis based on ultra-high pressure water cutting method," China Forest Products Industry (3), 29-32. DOI: 10.3969/j.issn.10015299.2016.03.007

GB/T 1031-2009 (2009). "Geometrical product specifications (GPS)-Surface texture: Profile method - Surface roughness parameters and their values," Standardization administration of China, Beijing, China.

He, J. L., Wang, Z., and Zhu, D. X. (2008). "Research and design on workpiece clamps for NC super high-pressure water jet cutting machines," Forestry Machinery \& Woodworking Equipment 36(5), 38-40. DOI: 10.3969/j.issn.2095-2953.2008.05.016

Ketan, V., Anandakrishnan, V., and Sathish, S. (2019). "Modelling and analysis of abrasive water jet machining of AA2014 alloy with $\mathrm{Al}_{2} \mathrm{O}_{3}$ abrasive using fuzzy logic," Materials Today: Proceedings. DOI: 10.1016/j.matpr.2019.06.733

Li, H. B. (2015). "Research on abrasive water jet cutting key technology," Wuhan University of Science and Technology.

Li, M. M., Cao, Y., Wang, Z., and Wang, Y. L. (2018a). "Optimization and analysis of processing parameters of wooden crafts based on ultra-high pressure water cutting method," Wood Research 63(1), 117-125.

Li, M. M., Xie, W. B., Wang, X. W., Cao, Y., and Wang, Z. (2018b). "Parameters optimization for ultrahigh-pressure pure water and abrasive water jet of Pterocarpus macarocarpus Kurz processing," Wood Research 63(5), 783-794.

Liu, H. C, Wang, Z. W, Cheng, M., Xu, W., Liu, H. J, Yu, X. C., and Zhi, S. J. (2018). "Development and application status of high pressure water jet cutting technology," Machine Tool \& Hydraulics 46(21), 173-179. DOI: 10.3969/j.issn.10013881.2018.21.038

Miao, X. J., Ye, F., Wu, M. P., Song, L., and Qiang, Z. R. (2019). “The method of 3D nozzle tilt cutting of abrasive water jet," The International Journal of Advanced Manufacturing Technology 103(5-8), 3109-3104. DOI: 10.1007/s00170-019-03757-4

Narayanan, C., Balz, R., Weiss, D. A., and Heiniger, K. C. (2013). "Modelling of abrasive particle energy in water jet machining," Journal of Materials Processing Technology 213(12), 2201-2210. DOI: 10.1016/j.jmatprotec.2013.06.020 
Pahuja, R., and Ramulu, M. (2019). "Study of surface topography in Abrasive Water Jet machining of carbon foam and morphological characterization using Discrete Wavelet Transform," Journal of Materials Processing Technology 273. DOI: 10.1016/j.jmatprotec.2019.05.030

Wang, Z., He, J. L., and Jiang, H. S. (2010). "Surface roughness of wood cut by extrahigh pressure water-jet," China Wood Industry 24(02), 38-40. DOI: 10.3969/j.issn.1001-8654.2010.02.012

Zhang, S. J., Chen, B., Wu, Y., and Chen, M. (2019). "Kinematic error compensation of a double swivel head in five-axis abrasive water jet machine tool," The International Journal of Advanced Manufacturing Technology 103(5-8), 2783-2793. DOI: 10.1007/s00170-019-03732-z

Article submitted: October 4, 2019; Peer review completed: January 14, 2020; Revised version received: February 1, 2020; Accepted: February 2, 2020; Published: March 23, 2020.

DOI: 10.15376/biores.15.2.3270-3277 